\title{
Cultivar-Dependent Expression of a Maize Lipoxygenase Responsive to Seed Infesting Fungi
}

\author{
Richard A. Wilson, ${ }^{1}$ Harold W. Gardner, ${ }^{2}$ and Nancy P. Keller ${ }^{1}$ \\ ${ }^{1}$ Department of Plant Pathology and Microbiology, TAMUS 2132, Texas A \& M University, College Station \\ 77843-2132, U.S.A.; ' $U S D A-A R S$, National Center for Agriculture Utilization Research, Peoria, IL 61604, \\ U.S.A. \\ Submitted 20 December 2000; Accepted 28 March 2001.
}

\begin{abstract}
Maize kernels are highly susceptible to Aspergillus spp. infection and aflatoxin (AF) contamination. Fatty acid signaling molecules appear to mediate the plant-fungal interaction by affecting the growth, development, and AF production of the fungus. In particular, fatty acid derivatives of the plant lipoxygenase (LOX) pathway are implicated in the Aspergillus spp.-seed interaction. The 9(S)hydroperoxide derivative of linoleic acid promotes transcription of AF genes, whereas the $13(S)$-hydroperoxide derivative decreases $\mathrm{AF}$ gene expression and production; both are sporulation factors. Our goal was to identify $L O X$ genes responsive to Aspergillus spp. colonization and determine their specificities, $9(S)$ - or $13(S)$-. Screening maize $L O X$ expressed sequence tags (ESTs) identified one clone, cssap 92, which is highly expressed in Aspergillus spp.infected seed susceptible to AF contamination and repressed in lines with resistance to AF contamination. The accumulation of cssap 92 transcript was similar during Fusarium spp. infection. The cDNA clone has $94 \%$ identity to the previously described $L 2$ LOX gene from maize. Product-specificity analysis of the CSSAP 92 protein shows that it preferentially adds oxygen to carbon 9 of linoleic acid. Because 9(S)-hydroperoxy linoleic acid has been implicated as an aflatoxin-signaling molecule, it is possible that cssap 92 could be used as a biomarker that is indicative of $\mathrm{AF}$ resistance in maize lines.
\end{abstract}

Additional keywords: Aspergillus flavus, Fusarium verticillioides, plant defense gene, Zea mays.

An intractable problem facing maize growers worldwide is contamination of crops by mycotoxins, particularly aflatoxin (AF), a potent carcinogen. During years with high temperatures and drought stress, the invasion of kernels by opportunistic seed infesting fungi of the Aspergillus genus, namely Aspergillus parasiticus and Aspergillus flavus, is prevalent (Payne 1992). These fungi readily produce AFB1, leading to substantial crop loss in developed countries and significant AF-associated health problems in those countries unable to implement detection and decontamination strategies.

Corresponding author: N. P. Keller; Current address: 882 Russell Labs, University of Wisconsin, Department of Plant Pathology, 1630 Linden Drive, Madison 53706, U.S.A.; Fax: +1-608-263-2626;

E-mail: npk@plantpath.wisc.edu implement detection and decontamination strategies. Because traditional plant protection and breeding methods are not sufficient to prevent this disease, research efforts have turned to deciphering the molecular events regulating the Aspergillus spp.-seed interaction as a means to develop effective control measures.

One research approach is the elucidation of genes and plant host gene products that might stimulate or repress Aspergillus spp. infection and/or AF contamination of seed. An emerging concept of the seed-Aspergillus spp.-AF interaction is the mediation of molecular cross-talk between the two organisms via fatty acid signaling. Burow et al. (1997) demonstrated that fatty acid derivatives of seed lipoxygenase (LOX) directly affect the production of AF in vitro. Specifically, LOX products $13(S)$-hydroperoxy-9(Z),11 (E)-octadecadienoic acid (13S-HPODE), and 13(S)-hydroperoxy-9(Z),11(E),15(Z)-octadecatrienoic acid (13S-HPOTE) reduced levels of AF gene transcripts and $9(S)$-hydroperoxy-10(E),12(Z)-octadecadienoic acid (9S-HPODE) extended the time period in which AF gene transcripts were detected. Downstream derivatives of $13 \mathrm{~S}$ HPODE and 13S-HPOTE, including methyl jasmonate (Goodrich-Tanrikulu et al. 1995) and various C6-C12 alkanal and alkenal volatiles (Doehlert et al. 1993; Zeringue et al. 1996) can also inhibit AF production. The effects of these compounds on $\mathrm{AF}$ production are distinct from their effects on fungal growth and development. In fact $9 S$ - and 13S-HPODE as well as linoleic and linolenic acid elicit asexual sporulation in Aspergillus spp., presumably by mimicking endogenous Aspergillus spp. sporogenic factors (Calvo et al. 1999). Furthermore, Burow et al. (2000) have shown that expression of a peanut $L O X$ gene, pnloxl, is stimulated by A. parasiticus infection and that levels of $9 S$-HPODE increase in Aspergillus spp.-infected peanut seed. Therefore, the fungus appears to receive morphological and chemical developmental signals from the host plant that are a direct consequence of fungal invasion.

Several lines of evidence suggest a significant role for the LOX pathway in plant-microbe interactions (Gardner 1991; Kolomiets et al. 2000; Kuhn and Thiele 1999). Lipoxygenases catalyze the addition of a peroxy group to either carbon 13 or 9 of the polyunsaturated linoleic and linolenic fatty acids. From these products, many metabolites are formed, including hydroxy fatty acids, derivatives of which have regulatory roles in plant metabolism. During wounding, methyl jasmonate and 
C6 volatiles derived from the LOX pathway (Bate and Rothstein 1998) act as diffusible signals that initiate the plant defense response (Bohlman et al. 1998). Other products of the LOX pathway are known to have, or be precursors of, molecules with antimicrobial-insecticidal properties. For example, in potatoes, the elimination of LOX activity by antisense technology was reflected in the weight gain of insect pests feeding on the antisense potatoes (Royo et al. 1999). Antisense suppression of a $L O X$ gene in tobacco allowed Phytophthora parasitica to successfully infect a resistant tobacco cultivar (Rance et al. 1998). In addition, LOX gene expression in plant tissues is spatially and temporally regulated and is often important for normal plant development (Dubbs and Grimes 2000a; Dubbs and Grimes 2000b; Eiben and Slusarenko 1994; Rodriguez-Concepcion and Beltran 1995; Van Mechelen et al. 1999; Wisniewski et al 1999).

Considering the importance of $L O X$ genes to plant development and defense and the putative role of LOX products in directing Aspergillus spp. developmental processes, our lab focuses on the characterization of $L O X$ genes involved in the seed-Aspergillus spp. interaction. Previously, we described a peanut seed $L O X$ gene responsive to Aspergillus spp. colonization that may be involved in the peanut seed-Aspergillus spp. interaction. Here, we describe a $L O X$ gene active in the maize-Aspergillus spp. interaction. As AF biosynthesis is confined primarily to colonized embryonic tissues of the maize kernel (Guo et al. 1996; Keller et al. 1994), the focus of this study was to identify Aspergillus spp. responsive LOX present in the maize embryo and to determine their product specificity (13S-HPODE or $9 S$-HPODE). An examination of nine putative expressed sequence tag (EST)-LOX clones resulted in the identification of two embryo-expressed $L O X$ genes. One of these, cssap 92, encodes a full-length LOX protein with high sequence identity to the previously described maize LOX protein, L2 (Jensen et al. 1997). The CSSAP 92 protein is a $9 S$-HPODE LOX whose transcript is expressed differentially in maize lines with different degrees of susceptibility to A. flavus infections.

\section{RESULTS}

\section{Sequencing the EST LOX clone cssap 92.}

Sequence comparisons among 21 putative maize EST LOX clones showed that nine were nonhomologous. Homologous clones are defined as having $>97 \%$ identity over the regions of overlap (sequences not shown). The nine $L O X$ clones were individually radiolabeled and used to probe total RNA that was extracted over a 48-h time course from Tx772-Fr2128 (resistant to AF) maize embryos that had been i) inoculated with A. flavus, ii) not inoculated but allowed to germinate, and iii) mock inoculated (control). Seven failed to hybridize to any transcripts, indicating these are not ESTs of embryoexpressed $L O X$ in this cultivar. The pattern of expression in the maize embryo for the remaining two clones, cssap 92 and chssh 76, are shown in Figure 1. Both putative $L O X$ genes are expressed in germinating tissues and the control but are absent (in the case of chssh 76) or present at reduced levels in embryos infected with A. flavus. This is seen most clearly when the cssap 92 cDNA clone is used as a probe. Identical results were achieved with the $3^{\prime}$ end of cssap 92 as a gene-specific probe for this transcript.
To ensure that cssap 92 and chssh 76 were unique $A$. flavusresponsive $L O X$ genes, the $3^{\prime}$ end of cssap 92 was radiolabeled and hybridized to the cssap 92 and chssh 76 cDNA clones. Available EST sequence data for the chssh 76 clone showed that it possessed the $3^{\prime}$ end of this gene. The $3^{\prime}$ fragment of cssap 92 does not cross-hybridize to chssh 76. Conversely, the radiolabeled $3^{\prime}$ end of chssh 76 hybridized to the chssh 76 cDNA clone but not to cssap 92 (data not shown). The 3' radiolabeled end of cssap 92 also was used to probe digested genomic DNA. In Southern hybridization analyses, the cssap 92 probe hybridized only to one band, suggesting that it was specific to cssap 92 (data not shown).

Because cssap 92 gave the strongest signal (Fig. 1), we concentrated on characterizing this gene. Sequence analysis of the $5^{\prime}$ and $3^{\prime}$ ends of the cssap 92 EST clone indicated that the EST clone probably contained the full-length transcript of cssap 92. Sequencing of the internal portion of cssap 92 showed that it appeared to encode for an allele of the previously published partial cDNA clone, $L 2$, of maize (Jensen et al. 1997). Over the overlap region, cssap 92 shares $94 \%$ identity with $L 2$ at the nucleotide and amino acid level (data not shown). The cssap 92 EST clone appears to be a full-length transcript and, relative to the partial $L 2$ clone, has an extra 179 codons, including a putative ATG start site determined by sequence homology with barley LOXI and confirmed by obtaining active protein translated from this codon (see below).

cssap 92 is expressed differentially in embryos of maize cultivars infected with Aspergillus flavus.

There is a delay in the induction of expression of cssap 92 and chssch76 in A. flavus-infected embryos of Tx772-Fr2128

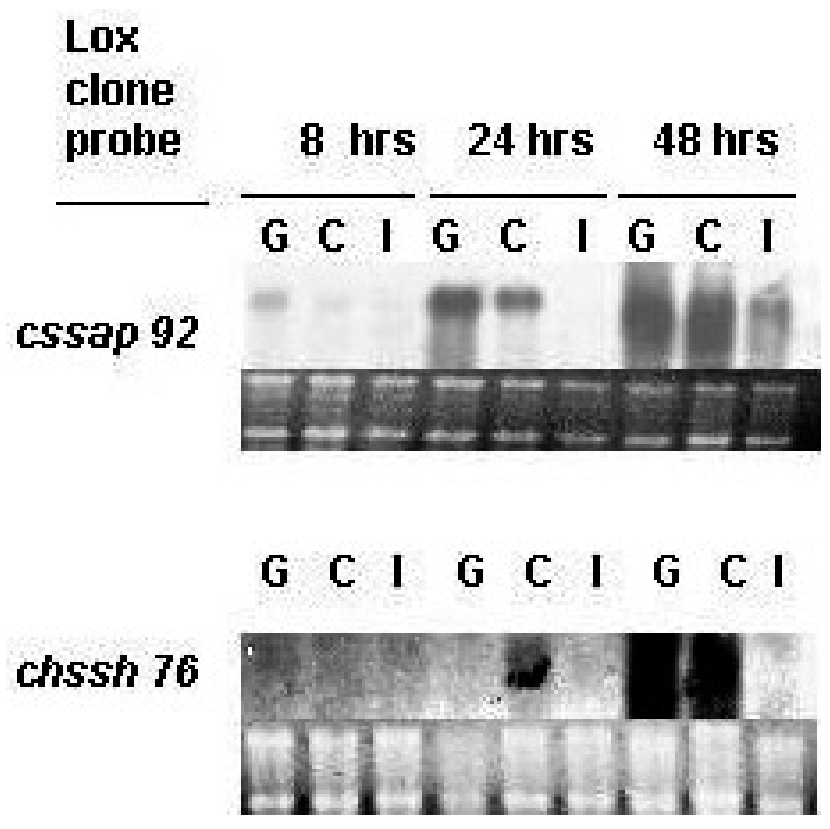

Fig. 1. Transcript analysis of two putative, embryo-expressed lipoxygenase $(L O X)$ genes with radiolabeled $L O X$ cDNA clones as probes. RNA was extracted from corn embryos of the backcross line Tx772-Fr2128 at the time points indicated. Total RNA $(5 \mu \mathrm{g})$ was loaded in each lane. $\mathrm{G}=\mathrm{RNA}$ from an uninfected, germinating embryo. $\mathrm{C}=$ Uninfected, nongerminating control. I = Embryonic RNA from corn infected with Aspergillus flavus 12$\mathrm{S}$. The experiment was repeated three times, and results shown are typical of three separate experiments. Lower panel shows ribosomal RNA stained with ethidium bromide to indicate loading of RNA samples. 
compared to the control embryos (Fig. 1). To investigate whether this dampening of $L O X$ expression (in comparison to control) was a conserved feature of the maize kernel response to Aspergillus spp. infections, we examined cssap 92 expression in several lines of maize, varying in degrees of susceptibility to Aspergillus spp. colonization and AF contamination. Figure 2 shows the expression of cssap 92 in the control and A. flavus-infected embryos of Asgrow 404 (Monsanto, St. Louis, MO, U.S.A.), a commercial seed line susceptible to AF contamination (J. Betran, personal communication). In this line, there are similar levels of cssap 92 expression in the control and infected embryos, up to $48 \mathrm{~h}$ postinoculation. At $48 \mathrm{~h}$ and beyond, however, cssap 92 expression increases dramatically in the infected embryos compared with the control. This is in contrast to the decreased cssap 92 expression in the infected embryos of Tx772-Fr2128 at $48 \mathrm{~h}$ (Fig. 1).

Figure 3 shows the expression of the cssap 92 gene at $48 \mathrm{~h}$ postinoculation with $A$. flavus in three maize lines that show some resistance to AF contamination (Campbell and White 1995; J. Betran, personal communication): Tex6, MASqK and Tx772, and the susceptible line Tx714. Once again, we see that the patterns of cssap 92 expression differs between the various maize lines. Although expression of cssap 92 in the control embryos does not show any consistent pattern between the resistant or susceptible lines, it is interesting to note that in infected embryos, a definite pattern can be seen. cssap 92 mRNA accumulation is observed in infected embryos of susceptible lines but is not expressed or reduced in message accumulation during infection of resistant lines at the time points examined.

\section{cssap 92 is expressed in root and stem tissue} of maize and induced by wounding and methyl jasmonate.

To examine organ-specific expression in healthy plants, cssap 92 mRNA accumulation was also examined in leaf, root, and stem tissues. Expression of cssap 92 transcripts was not detected in young leaves but, similar to L2 (Jensen et al. 1997), was detected in root tissue. In addition, cssap 92 expression was observed in stem tissue (data not shown).

An antiserum capable of recognizing L2 and L1 LOX isoforms was used (Jensen et al. 1997) to detect putative L2 pro-

\section{Time course (hrs)}

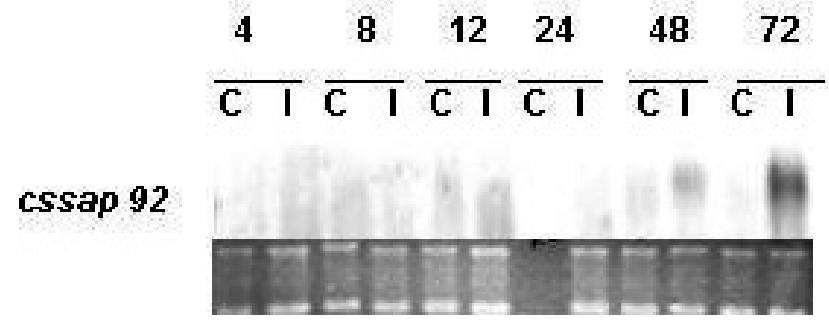

Fig. 2. RNA from control (C) and Aspergillus flavus-infected (I) embryos of Asgrow 404 seeds (Monsanto, St. Louis, MO, U.S.A.) was extracted at $4,8,12,24,48$, and $72 \mathrm{~h}$ time points. Total RNA (5 $\mu \mathrm{g})$ was loaded in each lane. RNA was probed with cssap 92. The experiment was repeated three times, and results shown are typical of three separate experiments. Lower panel shows ribosomal RNA stained with ethidium bromide to indicate loading of RNA samples. tein in maize tissues after treatment with methyl jasmonate. Because Jensen et al. (1997) used a different corn line in their studies (W64A), and we observed differential expression of cssap 92 in different lines in response to Aspergillus spp. infection, we examined the expression of cssap 92 after treatment of embryos with methyl jasmonate and wounding in Asgrow 404 and TX772-Fr2128. Our findings, similar to those of Jensen et al. (1997), showed that cssap 92 was expressed under all of these conditions in both lines (data not shown).

cssap 92 is expressed differentially in maize lines in response to Fusarium verticillioides.

Another serious mycotoxigenic fungus that frequently infects maize kernels is F. verticillioides (formerly called Fusarium moniliforme). To address the question of whether the expression of cssap 92 in response to A. flavus infection is a specific or general response to pathogen invasion, we studied the expression of this gene after inoculation with a strain of $F$. verticillioides isolated from maize. At $48 \mathrm{~h}$ postinoculation, the levels of expression of cssap 92 in Asgrow 404 is higher for A. flavus-infected seed than in $F$. verticillioides-infected seed (Fig. 4). cssap 92 is more highly expressed in A. flavusand $F$. verticillioides-infected seed than in uninfected control seed.

In Tx772-Fr2128, at 24 h postinoculation, cssap 92 expression is seen only in the uninfected control seed. At $48 \mathrm{~h}$ postinoculation, similar levels of cssap 92 expression are seen in all three seed treatments.

\section{In vitro expression of CSSAP 92 and analysis of its products.}

In order to determine the oxidation products of CSSAP 92, a full-length cDNA was expressed in a bacterial expression system. Protein extracts from bacteria containing pRAW2, the pET-30a expression vector containing the entire cssap 92 coding region, had measurable hydroperoxidizing activities at $\mathrm{pH}$ 6.5 to 7.5 (data not shown), and maximum activity was observed at $\mathrm{pH}$ 7.0. No activity was seen from extracts of bacteria containing only the pET-30a cloning vector or pRAW1 (encoding CSSAP 92 truncated at the $\mathrm{N}$ terminus) at this $\mathrm{pH}$ level. Activity at neutral $\mathrm{pH}$ suggests that the major product of

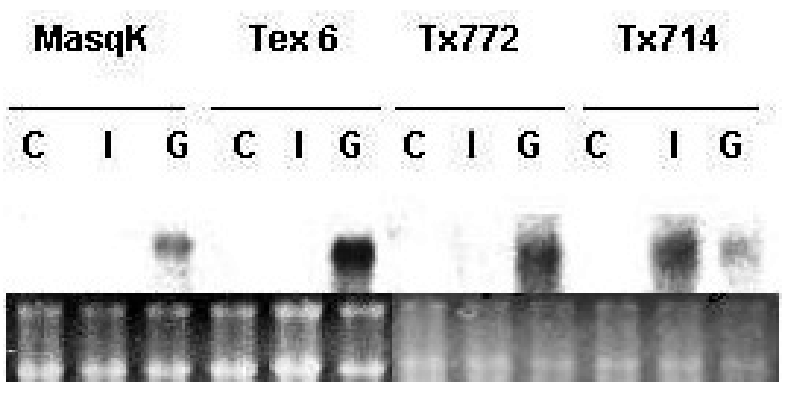

Fig. 3. The 0.26-kb 3' end of the cssap 92 gene was used to probe total RNA of embryos from corn lines MASqK, Tex 6, Tx772, and Tx714. Total RNA $(5 \mu \mathrm{g})$ was loaded in each lane. $\mathrm{C}=$ Uninfected, nongerminating control. $\mathrm{I}=$ Aspergillus flavus-infected. $\mathrm{G}=$ Germinating. RNA was extracted from corn embryos at a single time point, $48 \mathrm{~h}$ postinoculation. The experiment was repeated three times, and results shown are typical of three separate experiments. Lower panel shows ribosomal RNA stained with ethidium bromide to indicate loading of RNA samples. 
CSSAP 92 would be 9S-HPODE. In addition, the protein contains the bipartite Thr-Val (TV) motif, which was shown by Hornung et al. (1999) to specify $9 S$-HPODE production. Examination of the hydroperoxy linoleic acid products confirms that this is, indeed, the case because full-length CSSAP 92 protein produced $96 \%$ 9S-HPODE (Table 1). The small amount of $13 S$-HPODE that was present was largely racemic, with some tendency toward (R), indicating that some of the isomer arose from autoxidation or LOX-catalyzed autoxidation (Table 1$)$. The $(E, E)$-diene isomers arise from rearrangements of peroxyl radicals, which are generally racemic and were not examined for $(R, S)$-stereo configuration. Interestingly, the CSSAP 92 protein lacking the $\mathrm{N}$ terminus was weakly active at alkali $\mathrm{pH} 12.5$, suggesting that it would mainly produce $13 S$-HPODE. Indeed, $13 S$-HPODE is the major reaction product of the truncated protein at this $\mathrm{pH}$ level (Table 1), whereas the full-length clone was not active.

\section{Analysis of HPODE in vivo and AF production in two maize lines inoculated with $A$. flavus.}

To couple expression data of cssap 92 (and other LOX genes) with activity and product specificity of the protein in situ, a series of control and infected seeds (Asgrow 404 and Tx772-Fr2128) were examined for LOX activity at $0,12,24$, 48 , and $72 \mathrm{~h}$ postinoculation. The 9,10-ketol (analyzed as methyl 9,10-diOTMS derivative) was a major product in all cases (data not shown). The 9,10-ketol arises from allene oxide synthase (AOS) action on 9S-HPODE (Gardner et al. 1970). Trace amounts of 12,13-ketols derived from AOS action on $13 S$-HPODE were detected in some samples. Therefore, the LOX proteins present in the mature seed are mainly $9 S$-HPODE producers.

Although 9S-HPODE apparently is present in seed under all conditions, the cssap 92 transcript encoding a 9S-HPODE producer nonetheless increases during infection in AFsusceptible seed and is reduced in AF-resistant seed. We wanted to see whether this difference in cssap 92 expression correlated with measurable differences in the concentration of AF produced by A. flavus growing on AF-resistant or AFsusceptible lines. The amount of AF present in A. flavusinfected Asgrow 404 and Tx772-Fr2128 seed at 0, 24, 48, 72, and $120 \mathrm{~h}$ postinoculation is shown in Table 2 . AF was detected earlier ( $72 \mathrm{~h}$ ) in the Asgrow 404 seed, but by $120 \mathrm{~h}$, there was no significant difference in AF accumulation in the two lines.

\section{DISCUSSION}

AF contamination of oil seed crops by Aspergillus spp. is a grave concern for farmers in Africa, the Asian subcontinent, and the southern United States where conditions are often optimal for AF production (Payne 1992). One way to address this problem is to develop a better understanding of the molecular processes underlying the Aspergillus spp.-seed interaction. Studies in recent years have implicated a lipidmediated signaling process important in the Aspergillus spp.aflatoxin-seed interaction. Products of lipoxygenases, identified as having a role in plant defense against aggressive pathogens such as $P$. parasitica (Rance et al. 1998) and Pythium irregulare (Staswick et al. 1998), also might have a role in the Aspergillus spp.-seed-AF interaction problem. Studies by Burow et al. (1997) have shown that $13 S$-HPODE reduces the expression of AF gene transcripts, whereas 9S-HPODE increases the time during which these biosynthetic genes are expressed. Furthermore, derivatives of the 13S-LOX pathway have been shown to inhibit AF production in vitro (GoodrichTanrikulu et al. 1995; Zeringue et al. 1996). Recently, Burow et al. (2000) have shown that a $L O X$ gene in the mature peanut seed is expressed during infection by A. parasiticus, thus establishing the first case of an Aspergillus spp.-responsive LOX. This study aimed to characterize $L O X$ gene response and product specificity in Zea mays, another important oil seed crop highly susceptible to Aspergillus spp.-infection and AF contamination.

Transcript analysis indicated that at least two distinct $L O X$ genes respond to A. flavus colonization. We made the novel observation that one of these genes, cssap 92, is expressed differentially in maize lines showing different degrees of susceptibility to AF contamination (Figs. 1, 2, and 3). The corn lines Tx772-Fr2128, Tx722, Tex6, and MASqK showed low levels of expression of the cssap 92 transcript in infected embryos. These four lines show some resistance to AF in the field (Campbell and White 1995; J. Betran, personal communication). Asgrow404 and Tx714 are susceptible to AF contamination in the field and have increased expression of the cssap 92 transcript in infected embryos.

To determine whether cssap 92 expression was affected by different fungal pathogens, we infected Asgrow 404 (AF susceptible) and Tx772-Fr2128 (AF resistant) with the fumonisin-producing fungus $F$. verticillioides (Fig. 4). In both lines, cssap 92 had the same pattern of expression in response to both fungi. This suggests that the differential expression of cssap 92 that was seen in the different maize lines is a general mode of response to seed pathogens. In Asgrow 404, however, the level of gene expression was less during $F$. verticillioides infection than during A. flavus infection at the same time point. A future goal for our laboratory is to examine whether there is an interaction between cssap 92 expression, genetic background, and different fungal pathogens.

Sequencing the cssap 92 EST clone showed that the fulllength transcript is approximately $94 \%$ identical to $L 2$ at the

\section{Asgrow $404 \quad$ Tx772i Fr2128}

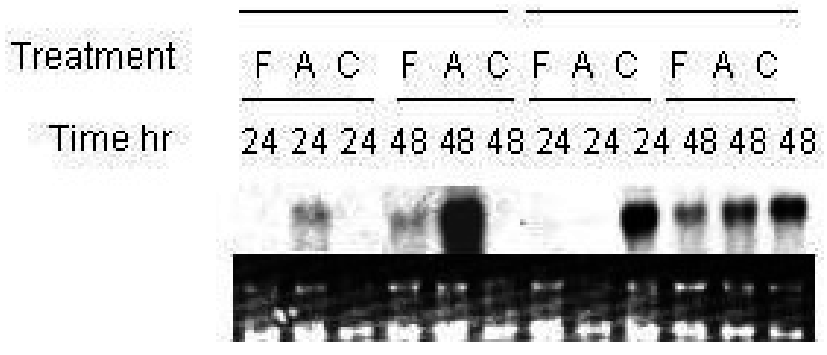

Fig. 4. Asgrow 404 (Monsanto, St. Louis, MO, U.S.A.) and Tx772Fr2128 seeds were challenged with Fusarium verticillioides (F), Aspergillus flavus (A), and a water control (C). RNA was extracted from the embryos of these seeds at 24 and $48 \mathrm{~h}$ postinoculation. Total RNA $(5 \mu \mathrm{g})$ was loaded in each lane. RNA was probed with the $0.26-\mathrm{kb} 3^{\prime}$ end of the cssap 92 gene. The experiment was repeated three times, and results shown are typical of three separate experiments. Lower panel shows ribosomal RNA stained with ethidium bromide to indicate loading of RNA samples. 
nucleotide level (results not shown). This suggests that cssap 92 is a possible allelic variant of $L 2$, which is thought to be a $9 S$ HPODE producing LOX, based on the crude extract examination of corn seed (Gardner 1970) and amino acid sequence. Both proteins have the TV motif (Hornung et al 1999) to determine 9S-HPODE specificity. To accurately determine the nature of the product specificity of CSSAP 92, the protein was purified and reacted with linoleic acid. The purified CSSAP 92 protein has a $\mathrm{pH}$ optimum of 7.0, indicative of type 2 LOX (Shibata 1996). Product analysis confirmed that cssap 92 produced mainly $9 S$-HPODE (96\%). Intriguingly, an $\mathrm{N}$-terminal truncated version of this protein, PRAW1, has very low but measurable activity at alkaline $\mathrm{pH}$ levels and produces mainly $13 S$-HPODE. From the structure of soybean LOX-1, it has been determined that the N-terminal region of the protein forms a beta-barrel structure (Boyington et al. 1997; Prigge et al. 1997) shown to target lipid body LOX to liposomes and lipid bodies in cucumber seedlings (May et al 2000). Without structural studies, one can only speculate as to why deleting this region would affect the specificity of CSSAP 92 in vitro. With $13 S$-specific LOXs, $\mathrm{H}$-removal and $\mathrm{O}_{2}$ insertion occurs on opposite sides of the substrate to $9 S$-specific LOXs (Funk et al. 1987; Lehman 1994). At high $\mathrm{pH}$ levels, the substrate molecule enters the active site in an orientation that favors $13 S$-HPODE production, presumably because the carboxylate anion prevents its insertion into the hydrophobic pocket of LOX in the inverse orientation. As the $\mathrm{pH}$ is lowered, the molecule reverses its orientation, leading to more 9S-HPODE production (Gardner 1989). Deleting the Nterminal residues of CSSAP 92 could lead to a change in protein conformation and activity such that PRAW1 is active only at high $\mathrm{pH}$ levels. At elevated $\mathrm{pH}$ levels, the substrate molecule might enter the active site only in an orientation that leads to $13 S$-HPODE production.

We predicted that the differences in cssap 92 transcript levels (and possibly the amount of 9S-HPODE) in Asgrow 404 and Tx772-Fr2128 kernels could be reflected by differences in mycotoxin production. Table 2 shows that Asgrow 404 seeds have measurable quantities of $\mathrm{AF}$ at earlier time points $(72 \mathrm{~h})$ than seeds of Tx772-Fr2128. Because these lines are not isogenic, this difference could be the result of differences in how A. flavus grows and develops on different maize seeds. Whatever the reason, it is clear that Asgrow 404 is contaminated with more AF per gram of embryo at earlier time points than Tx772-Fr2128. This is consistent with the earlier appearance of the cssap 92 transcript in Asgrow 404. At $120 \mathrm{~h}$ postinoculation, the AF level in both lines is very similar, suggesting that cssap 92 expression in Tx772-Fr2128 is no longer delayed and contributes to AF production in the seed. To definitively make this conclusion, we need to compare $\mathrm{AF}$ production in corn lines that are isogenic to Asgrow 404 but deleted in cssap 92. The fact that Asgrow 404 expresses cssap 92 under infection conditions affords the potential for increased formation of 9S-HPODE and its major metabolite, 9,10-ketol. Although 9S-HPODE increases AF gene expression, the effects of 9,10-ketol on AF production are not known. Nevertheless, these metabolites may explain increased AF gene expression in vitro. The differential expression of cssap 92 could explain why in laboratory and field situations, Asgrow 404 exhibits more susceptibility to AF contamination than does Tx772-Fr2128.

In summary, we believe to have uncovered a potential role for the cssap 92 gene encoding a $9 S$-HPODE producing LOX in its contribution toward AF contamination. Expression of cssap 92 clearly responds to A. flavus infestation. Moreover, its expression is also different in resistant and susceptible maize lines, with expression strongest during infection of susceptible lines. Because this work was performed under laboratory conditions, it most closely resembles the reaction of postharvest seeds to colonization during storage conditions. In future studies, we would like to examine the expression of cssap 92 as a biomarker denoting resistance or susceptibility to AF in the field. Because cssap 92 also responds to infection by $F$. verticillioides, it would be interesting to determine whether $L O X$ expression plays a role in the Fusarium spp.maize interaction.

\section{MATERIALS AND METHODS}

\section{EST- $L O X$ clones.}

Twenty-one EST cDNA pSPORT (Promega, Madison, WI, U.S.A.) clones encoding putative $L O X$ genes from a number of different maize libraries were received from J. Duvick of Pioneer Hi-Bred International (Des Moines, IA, U.S.A.). Sequence data of the $5^{\prime}$ and $3^{\prime}$ ends facilitated comparisons of all clones. The Sequencher software program (Gene Codes, Ann Arbor, MI, U.S.A.) allowed the determination of homologous and nonhomologous clones on the basis of the available sequence, with homologous clones determined as having $>97 \%$ identity over the overlap region.

\section{Sequencing and sequence analysis.}

Sequencing the full-length $L O X$ cDNA clone cssap 92 was performed with the automated sequencing service provided by the Gene Technologies Department at Texas A \& M University. The nucleotide sequence of both strands was determined. Sequences were aligned with Sequencher software. The fulllength cssap 92 sequence was compared with known LOX genes in the GenBank database with the BLAST program.

Table 1. Analysis of regio and stereo configurations of hydroperoxy-9(Z), 11(E)-octadecadienoic acid (HPODEs) produced by a full-length CSSAP 92 (pRAW2 construct) as their methyl HODEs at $\mathrm{pH} 7.0$ and by a truncated CSSAP 92 (pRAW1 construct) at $\mathrm{pH} 12.5^{\mathrm{a}}$

\begin{tabular}{|c|c|c|c|c|c|c|}
\hline \multirow[b]{3}{*}{ Extract } & \multicolumn{3}{|c|}{ 13-HODEs (\% of total) } & \multicolumn{3}{|c|}{ 9-HODEs (\% of total) } \\
\hline & \multirow{2}{*}{$\begin{array}{c}9(Z), 11(E) \text {-diene } \\
13(S)\end{array}$} & \multicolumn{2}{|c|}{$9(E), 11(E)$-diene } & \multicolumn{2}{|c|}{$10(E), 12(Z)$-diene } & \multirow{2}{*}{$\begin{array}{c}10(E), 12(E) \text {-diene } \\
9(R, S)\end{array}$} \\
\hline & & $13(R)$ & $13(R, S)$ & $9(S)$ & $9(R)$ & \\
\hline pRAW2 (pH 7.0) & 0.27 & 0.65 & 0.63 & 95.7 & 0.4 & 2.4 \\
\hline pRAW1 (pH 12.5) & 50.7 & 12.1 & 8.7 & 11.6 & 10.3 & 6.6 \\
\hline
\end{tabular}

${ }^{a}$ Incubations of linoleic acid with pRAW1 and pET30a extracts gave only trace amounts of HPODEs at pH 7.0. pRAW2 extracts produced only trace amounts of HPODEs at $\mathrm{pH} 12.5$. 


\section{Plant materials and treatments.}

The maize cultivars used in this study were Asgrow 404 and Tx714, lines that are highly susceptible to AF in the field, and Tx772-Fr2128, Tex6, MASqK, and Tx772, lines that have shown some resistance to AF contamination in the field (J. Betran, personal communication). Asgrow 404 is a commercial hybrid. Tx772, Tx714, and Tex6 are inbred lines. Tx772Fr2128 is a hybrid of the two inbred lines Tx 772 and Fr2128. MASqK is a derived line from the MASqK population, developed in Georgia. Prior to treatment, kernels were sterilized by immersion in 10\% Clorox (Oakland, CA, U.S.A.) for $3 \mathrm{~min}$, followed by immersion in sterile water for $3 \mathrm{~min}$. Seeds were inoculated with either A. flavus strain 12-S (provided by P. Cotty, USDA-ARS, New Orleans, LA, U.S.A.) or a $F$. verticillioides isolate (provided by T. Isakeit, Department of Plant Pathology and Microbiology, Texas A \& M). To sterilize, kernels were immersed for $30 \mathrm{~min}$ in $0.1 \%$ Tween 80 containing $10^{6}$ spores per $\mathrm{ml}$. Control kernels were immersed in Tween water for $30 \mathrm{~min}$. Neither Tween 80 or 20 affected $L O X$ gene expression (data not shown). Corn kernels were wounded with a sterile needle, piercing near the center of the embryo to a depth of approximately $1 \mathrm{~mm}$. To determine the effects of methyl jasmonate on $L O X$ gene expression, corn kernels were treated with a racemic mixture (Bedoukin Research, Danbury, CT, U.S.A.), as previously described for peanut seed (Burow et al. 2000). All treated and control seed were incubated in the dark at $30^{\circ} \mathrm{C}$ in petri dishes containing moist filter paper and a water reservoir. Samples were collected at the indicated time points.

For all RNA studies, only the maize embryo was used. The embryo was removed by wrapping the seed in the folds of a paper towel, striking carefully with a hammer to crack the kernel. The endosperm, aleurone layer, and scutellum were discarded, and the embryo was frozen in liquid nitrogen. Embryos were stored at $-80^{\circ} \mathrm{C}$.

\section{RNA and DNA extraction.}

RNA was isolated from kernel embryos, leaf, root, and stem with Trizol reagent (GIBCO-BRL, Rockville, MD, U.S.A.), following the manufacturer's protocol with modifications. Two embryos (approximately $150 \mathrm{mg}$ ) were used per extraction or $150 \mathrm{mg}$ of leaf, root, and stem, which were lyophilized overnight and ground in liquid nitrogen. Trizol $(1 \mathrm{ml})$ was added, the resulting aqueous phase was extracted with phenol-chloroform and chloroform-isoamyl alcohol, and the RNA was precipitated with isopropyl alcohol. The RNA was resuspended in water and reprecipitated in $8 \mathrm{M} \mathrm{LiCl}$, followed by a second reprecipitation in $3 \mathrm{M} \mathrm{NaOAc}$ and resuspension in $22 \mu \mathrm{l}$ of water. All solutions were prepared with diethyl pyrocarbonate-treated water.

Genomic DNA was isolated from young leaf tissue following the urea extraction procedure of Chen and Dellaporta (1993). Two grams of leaf was used per $6 \mathrm{ml}$ of lysis buffer.

\section{Northern blot analysis.}

RNA $(5 \mu \mathrm{g})$ was separated on a $1.2 \%$ agarose- $1.5 \%$ formaldehyde gel and transferred to Hybond membrane (Amersham Pharmacia Biotech, Piscataway, NJ, U.S.A.). Following prehybridization and addition of the probe, the membranes were hybridized overnight at $60^{\circ} \mathrm{C}$ and washed with increasing stringency, up to $0.1 \times \mathrm{SSC}(1 \times \mathrm{SSC}$ is $0.15 \mathrm{M} \mathrm{NaCl}$ plus
$0.015 \mathrm{M}$ sodium citrate) and $0.1 \%$ sodium dodecyl sulfate, at $60^{\circ} \mathrm{C}$.

Probes were obtained from the EST clones by releasing the $L O X$ inserts from the pSPORT1 cDNA cloning vector with the directional cloning restriction sites $\mathrm{SalI}$ (at the $5^{\prime}$ end) and NotI (at the $3^{\prime}$ end). The inserts were labeled with ${ }^{32} \mathrm{P}$ by the random primer method (Sambrook et al. 1989). To analyze cssap 92 expression, digestion of the cssap 92 cDNA clone with SalI and NotI yielded two fragments: a 234-bp SalI fragment from the $5^{\prime}$ end of the gene and an approximately $2.6-\mathrm{kb}$ SalI-NotI fragment. Both fragments were purified, radiolabeled, and used as probes in the RNA analysis. To confirm that the 2.6-kb SalI-NotI fragment was hybridizing only to the cssap 92 transcript, RNA blots were stripped and probed with an approximately $0.2-\mathrm{kb} K p n \mathrm{I}-N o t \mathrm{I}$ fragment containing the more variable $3^{\prime}$ untranslated regions (UTR) end of the cssap 92 gene.

\section{Southern blot analysis.}

Maize genomic DNA $(2 \mu \mathrm{g})$ were digested with EcoRI, HindIII, or XbaI and separated on an $0.8 \%$ agarose gel by electrophoresis. The DNA was transferred to Hybond membranes after denaturation in $0.4 \mathrm{M} \mathrm{NaOH}$ solution. Membranes were probed with the $0.2-\mathrm{kb} K p n \mathrm{I}-N o t \mathrm{I}$ fragment containing the $3^{\prime}$ UTR of the cssap 92 gene in conditions described above for RNA blots.

\section{Expression of the cssap $92 \mathrm{LOX}$ gene in Escherichia coli.}

The full-length cssap 92 cDNA clone was ligated into the expression vector pET-30a (Novagen, Madison, WI, U.S.A.). This was achieved by isolating a 2.6-kb SalI-NotI fragment of cssap 92, removing the SalI-NotI fragment from the pET-30a vector, and subcloning the cssap 92 fragment directly into pET-30a to give pRAW1. pRAW1 encodes for $2.6 \mathrm{~kb}$ of the cssap 92 and lacks approximately $0.24 \mathrm{~kb}$ of the $5^{\prime}$ coding region, including the putative start site. Next, approximately $0.3 \mathrm{~kb}$ of the $5^{\prime}$ end of cssap 92 was amplified from the pSPORT1 vector with $p f u$ polymerase (Stratagene, La Jolla, CA, U.S.A.) to introduce a unique NcoI site at the ATG codon. The polymerase chain reaction (PCR) product and pRAW1 were digested with $\mathrm{NcoI}$ and SalI, and the PCR product was ligated into pRAW1 to give pRAW2. Plasmid pRAW2 encodes the full-length cssap 92 coding region, in-frame with the pET-30a start site, preceded by the oligomeric histidine

Table 2. Aflatoxin concentration was measured with the indirect competitive enzyme-linked immunosorbent assay method for embryos extracted from Asgrow 404 (Monsanto, St. Louis, MO, U.S.A.) and Tx772-Fr2128 seeds contaminated with Aspergillus flavus ${ }^{\mathrm{a}}$

\begin{tabular}{lcc}
\hline Sample & Hours postinoculation & AF concentration \\
\hline Tx772-Fr2128 & 12 & 0 \\
24 & 0 \\
48 & 0 \\
72 & 0 \\
120 & $32.8 \pm 8.8$ \\
& 12 & 0 \\
Asgrow 404 & 44 & 0 \\
& 78 & 0 \\
& 120 & $10.4 \pm 3.1$ \\
& & $30.3 \pm 3.4$ \\
\hline
\end{tabular}

${ }^{a}$ Amounts are given in ppm. 
purification tag. pRAW1 and pRAW2 were maintained in DH5 $\alpha$ E. coli cells and transformed into the BL21 (DE3) strain of E. coli for induction of the CSSAP 92 protein. Induction was carried out following previously described protocols (Steczko et al. 1992). The best results were obtained when cells were incubated at $15^{\circ} \mathrm{C}$ for $20 \mathrm{~h}$, with $300 \mathrm{rpm}$ shaking and $1 \mathrm{mM}$ isopropyl- $\beta$-D-thiogalactopyranoside.

Analysis of the oxidation products of pRAW2 and pRAW1.

Total protein was extracted from bacteria containing pRAW1, pRAW2, and the pET-30a vector as described previously (Steczko et al. 1992). LOX activity of the extracts was assayed spectrophotometrically. Extracts were stored in dry ice prior to incubation with linoleic acid. Protein extracts were thawed and an aliquot diluted in $24 \mathrm{ml}$ of $50 \mathrm{mM} \mathrm{N}-2$ hydroxyethylpiperazine- $N^{\prime}$-2-ethanesulfonic acid (HEPES), $\mathrm{pH} 7.0$ and $50 \mu \mathrm{l}$ of $48 \mathrm{mM}$ methanolic linoleic acid at $25^{\circ} \mathrm{C}$ (final $\mathrm{pH}$ of 6.9). The solution was stirred magnetically in a 125-ml Erlenmeyer flask for $15 \mathrm{~min}$, after which time the $\mathrm{pH}$ level of the reaction was adjusted to 4.0 with $1 \mathrm{M}$ oxalic acid. $\mathrm{CHCl}_{3}-$ methanol $(75 \mathrm{ml}, 2: 1$, vol/vol) was added immediately to extract the products. The hydroperoxide derivatives contained in the bottom $\mathrm{CHCl}_{3}$ layer were analyzed for positional and stereochemical properties, as described previously (Gardner et al. 1998).

\section{Analysis of the oxidation products of endogenous maize LOXs.}

Maize seeds from Asgrow 404 and Tx772-Fr2128 lines were inoculated with A. flavus or mock inoculated and ground in a coffee grinder. Four maize seeds per time point were combined in each case. HEPES (50 mM, pH 7.0) was added at a rate of $10 \mathrm{ml}$ of maize meal per gram and the slurry homogenized for $1 \mathrm{~min}$ at full speed. After centrifugation at $10,000 \mathrm{~g}$ for $20 \mathrm{~min}$, the fat layer was aspirated and a $0.25 \mathrm{ml}$ aliquot of supernatant was stored at $-20^{\circ} \mathrm{C}$ for protein concentration determination. The remainder of the supernatant was added to a 125-ml Erlenmeyer flask, followed by the addition of HEPES buffer ( $\mathrm{pH}$ 7.0) to give a maize extract-HEPES buffer ratio of $1: 1.5(\mathrm{vol} / \mathrm{vol})$. The solution was stirred magnetically at a constant temperature of $25^{\circ} \mathrm{C}$. The substrate solution contained $140 \mathrm{mg}$ of linoleic acid in $5 \mathrm{ml}$ of methanol and was added by injection to the maize-HEPES buffer mix at a rate of $5 \mu \mathrm{l}$ of methanolic linoleic acid solution per $5 \mathrm{ml}$ of maize-HEPES mix to give a final concentration of $0.1 \mathrm{mM}$ linoleic acid. After $15 \mathrm{~min}$ of incubation at $25^{\circ} \mathrm{C}$ with stirring, the reaction was stopped by adjusting the $\mathrm{pH}$ to 4.0 with oxalic acid and extracting the products with a volume of $\mathrm{CHCl}_{3}-$ methanol $(2: 1 \mathrm{vol} / \mathrm{vol})$, which is equivalent to three times the volume of the reaction mix. The $\mathrm{CHCl}_{3}$ extract could be saved at $-20^{\circ} \mathrm{C}$ until product analysis. Products were analyzed by gas chromatography mass spectrometry, as described in Burow et al. (2000), and the $\alpha$-ketol products were examined as methyl ester-trimethylsilyloxy derivatives after $\mathrm{NaBH}_{4}$ reduction, giving two separable diastereoisomers for each $\alpha$-ketol, e.g., two separable methyl di-9,10-trimethylsilyloxy-12(Z)octa-decenoates.

\section{AFB1 quantitation.}

Maize seeds from Asgrow 404 and Tx772-Fr2128 lines inoculated with A. flavus were ground under liquid nitrogen with a mortar and pestle and transferred to a 1.5-ml Eppendorf tube. The sample was weighed and $500 \mu \mathrm{l}$ of $70 \% \mathrm{MeOH}$ was added. The sample was vortexed vigorously, spun briefly in a centrifuge, and left to sit overnight at room temperature in the dark. Following a brief vortex and centrifugation, an aliquot of the $\mathrm{MeOH}$ layer was diluted 1:5 with $0.01 \mathrm{M}$ phosphatebuffered saline and $50 \mu \mathrm{l}$ of the diluted samples were used for enzyme-linked immunosorbent assay (ELISA) measurements. AFB1 concentration was determined by indirect competitive ELISA with standards of known AFB1 concentrations. Treatments were performed in triplicate and analyzed with the SAS statistical software package (SAS Institute, Cary, NC, U.S.A.).

\section{ACKNOWLEDGMENTS}

We gratefully thank J. Duvick and J. Maddox of Pioneer Hi-Bred International for supplying the lox EST clones. We also thank J. Betran of Texas A \& M University for supplying the corn kernels, M. Grove for providing fatty acid analyses, and M. Kolomiets for helpful comments and discussion. This work is supported by a TxGrain grant and the Texas Corn Producers Board to R. A. Wilson and N. P. Keller.

\section{LITERATURE CITED}

Bate, N. J., and Rothstein, S. J. 1998. C6-volatiles derived from the lipoxygenase pathway induce a subset of defense-related genes. Plant J. 16:561-569.

Bohlmann, H., Vignutelli, A., Hilpert, B., Miersch, O., Wasternack, C., and Apel, K. 1998. Wounding and chemicals induce expression of the Arabidopsis thaliana gene Thi2.1, encoding a fungal defense thionin, via the octadecanoid pathway. FEBS Lett. 437:281-286.

Boyington, J. C., Gaffney, B. J., and Amzel, L. M. 1997. The three dimensional structure of soybean lipoxygenase-1: An arachidonic acid 15-lipoxygenase. Adv. Exp. Med. Biol. 400A:133-138.

Burow, G. B., Nesbitt, T. C., Dunlap, J., and Keller, N. P. 1997. Seed lipoxygenase products modulate Aspergillus mycotoxin biosynthesis. Mol. Plant-Microbe Interact. 10:380-387.

Burow, G. B., Gardner, H. W., and Keller, N. P. 2000. A peanut seed lipoxygenase responsive to Aspergillus colonization. Plant Mol. Biol. 42:689-701.

Calvo, A., Hinze, L., Gardner, H. W., and Keller, N. P. 1999. Sporogenic effect of polyunsaturated fatty acids on Aspergillus spp. development. Appl. Environ. Microbiol. 65:3668-3673.

Campbell, K. W., and White, D. G. 1995. Evaluation of corn genotypes for resistance to Aspergillus ear rot, kernel infection, and aflatoxin production. Plant Dis. 79:1039-1045.

Chen, L., and Dellaporta, S. 1993. Urea-based plant DNA miniprep. Pages 541-545 in: The Maize Handbook. M. Freeling and V. Walbot, eds. Springer Verlag, New York.

Doehlert, D. C., Wicklow, D. T., and Gardner, H. W. 1993. Evidence implicating the lipoxygenase pathway in providing resistance to soybean against Aspergillus flavus. Phytopathology 83:1473-1477.

Dubbs, W. E., and Grimes, H. D. 2000a. Specific lipoxygenase isoforms accumulate in distinct regions of soybean pod walls and mark a unique cell layer. Plant Physiol. 123:1269-1280.

Dubbs, W. E., and Grimes, H. D. 2000b. The mid-pericarp cell layer in soybean pod walls is a multicellular compartment enriched in specific lipoxygenase isoforms. Plant Physiol. 123:1281-1288.

Eiben, H. G., and Slusarenko, A. J. 1994. Complex and spatial and temporal expression of lipoxygenase genes during Phaseolus vulgaris (L.) development. Plant J. 5:123-135.

Funk, M. O., Jr., Andre, J. C., and Otsuki, T. 1987. Oxygenation of trans polyunsaturated fatty acids by lipoxygenase reveals steric features of the catalytic mechanism. Biochemistry 2621:6880-6884.

Gardner, H. W. 1970. Lipoxygenase from Zea mays: 9-D-hydroperoxytrans-10,cis-12-octadecanoic acid from linoleic acid. Lipids 5:678683.

Gardner, H. W. 1989. Soybean lipoxygenase-1 enzymically forms both $(9 S)$ - and (13S)-hydroperoxides from linoleic acid by a $\mathrm{pH}$-dependent 
mechanism. Biochim. Biophys. Acta 1001:274-281.

Gardner, H. W. 1991. Recent investigations into the lipoxygenase pathway of plants. Biochim. Biophys. Acta 1084:221-239.

Gardner, H. W., Grove, M. J., and Keller, N. P. 1998. Soybean lipoxygenase is active on nonaqueous media at low moisture: A constraint to xerophilic fungi and aflatoxins? J. Am. Oil Chem. Soc. 75:1801-1808.

Goodrich-Tanrikulu, M., Mahoney, N., and Rodriquez, S. B. 1995. The plant growth regulator methyl jasmonate inhibits aflatoxin production by Aspergillus flavus. Microbiology 141:2831-2837.

Guo, B. Z., Russin, J. S., Brown, R. L., Cleveland, T. E., and Widstrom, N. W. 1996. Resistance to aflatoxin contamination in corn is influenced by relative humidity and kernel germination. J. Food Prot. 59:276-281.

Hornung, E., Walther, M., Kuhn, H., and Feussner, I. 1999. Conversion of cucumber linoleate 13-lipoxygenase to a 9-lipoxygenating species by site-directed mutagenesis. Proc. Natl. Acad. Sci. USA 96:4192-4197.

Jensen, A. B., Poca, E., Rigaud, M., Freyssinet, G., and Pages, M. 1997. Molecular characterization of L2 lipoxygenase from maize embryos. Plant Mol. Biol. 33:605-614.

Keller, N. P., Butchko, R. A. E., Sarr, B., and Phillips, T. D. 1994. A visual pattern of mycotoxin production in maize kernels by Aspergillus spp. Phytopathology 84:483-488.

Kolomiets, M. V., Chen, H., Gladon, R. J., Braun, E. J., and Hannapel, D. J. 2000. A leaf lipoxygenase of potato induced specifically by pathogen infection. Plant Physiol. 124:1121-1130.

Kuhn, H., and Thiele, B. J. 1999. The diversity of the lipoxygenase family. FEBS Lett. 449:7-11.

Lehmann, W. D. 1994. Regio- and stereochemistry of the dioxygenation reaction catalyzed by (S)-type lipoxygenases or by the cyclooxygenase activity of prostaglandin $\mathrm{H}$ synthases. J. Biol. Chem. 242:5329-5335.

May, C., Hohne, M., Gnau, P., Schwennesen, K., and Kindl, H. 2000. The N-terminal beta-barrel structure of lipid body lipoxygenase mediates its binding to liposome and lipid bodies. Eur. J. Biochem. 267:1100-1109.

Payne, G. A. 1992. Aflatoxin in maize. Crit. Rev. Plant Sci. 10:423-440.

Prigge, S. T., Boyington, J. C., Faig, M., Doctor, K. S., Gaffney, B. J., and Amzel, L. M. 1997. Structure and mechanism of lipoxygenases.
Biochimie 79:629-636.

Rance, I., Fournier, J., and Esquerre-Tugaye, M.-T. 1998. The incompatible interaction between Phytophthora parasitica var. nicotianae race 0 and tobacco is suppressed in transgenic plants expressing antisense lipoxygenase sequences. Proc. Natl. Acad. Sci. USA 95:65546559.

Rodriquez-Concepcion, M., and Beltran, J. P. 1995. Repression of the pea lipoxygenase gene loxg is associated with carpel development. Plant Mol. Biol. 27:887-899.

Royo, J., Leon, J., Vancanneyt, G., Albar, J. P., Rosahl, S., Ortego, F., Castanera, P., and Sanchez-Serrano, J. J. 1999. Antisense-mediated depletion of a potato lipoxygenase reduces wound induction of proteinase inhibitors and increases weight gain of insect pests. Proc. Natl. Acad. Sci. USA. 96:1146-1151.

Sambrook, J., Fritsch, E. F., and Maniatis, T. 1989. Molecular Cloning: A Laboratory Manual, 2nd ed. Cold Spring Harbor Laboratory, Cold Spring Harbor, NY, U.S.A.

Shibata, D. 1996. Plant lipoxygenase genes. Pages 39-55 in: Lipoxygenase and Lipoxygenase Pathway Enzymes. AOCS Press, Champaign, IL, U.S.A.

Staswick, P. E., Yuen G. Y., and Lehman, C. C. 1998. Jasmonate signaling mutants of Arabidopsis are susceptible to the soil fungus Pythium irregulare. Plant J. 15:747-754.

Steczko, J., Donoho, G. A., Dixon, J. E., Sugimoto, T., and Axelrod, B. 1991. Effect of ethanol and low-temperature culture on expression of soybean lipoxygenase-1 in Escherichia coli. Prot. Express Pur. 2:221227.

Van Mechelen, J. R., Shuurink, R. C., Smits, M., Graner, A., Douma, A. C., Sedee, N. J. A., Schmitt, N. F., and Valk, B. E. 1999. Molecular characterization of two lipoxygenases from barley. Plant Mol. Biol. 39:1283-1298.

Wisniewski, J. P., Gardner, C. D., and Brewin, N. J. 1999. Isolation of lipoxygenase cDNA clones from pea nodule mRNA. Plant Mol. Biol. 39:775-783.

Zeringue, H., Brown, R., Neucere, J., and Cleveland, T. 1996. Relationships between C-6-C-12 alkanal and alkenal volatile contents and resistance of maize genotypes to Aspergillus flavus and aflatoxin production. J. Agric. Food Chem. 44:403-407. 